Pacific Journal of Mathematics

SELF-ADJOINT MULTI-POINT BOUNDARY VALUE 


\title{
SELF-ADJOINT MULTI-POINT BOUNDARY VALUE PROBLEMS
}

\author{
W. S. LOUD
}

It is the purpose of this paper to consider ordinary differential operators which are associated with multi-point boundary value problems on a finite interval. We do not consider singular boundary-value problems. Our interest is to determine just what multi-point boundary conditions are to be imposed if self-adjointness is to prevail.

In a recent paper [2] J. W. Neuberger has shown that a threepoint boundary value problem for a real second-order linear differential equation is not self-adjoint when the boundary conditions have the form

$$
\begin{aligned}
& a_{11} x(a)+a_{12} x^{\prime}(a)+a_{13} x(b)+a_{14} x^{\prime}(b)+a_{15} x(c)+a_{16} x^{\prime}(c)=0 \\
& \\
& \quad(a<b<c) \\
& a_{21} x(a)+a_{22} x^{\prime}(a)+a_{23} x(b)+a_{24} x^{\prime}(b)+a_{25} x(c)+a_{26} x^{\prime}(c)=0 .
\end{aligned}
$$

This is done by showing that the associated Green's function is never hermitian symmetric unless all four of $a_{13}, a_{14}, a_{23}$ and $a_{24}$ are zero, so that the third point $b$ does not enter into the boundary conditions.

Neuberger's results have been extended to higher-order problems by A. Zettl in [5].

It will appear that for a multi-point boundary-value problem to be self-adjoint the Green's function will have to be discontinuous in both its variables at the interior boundary points as well as at $t=s$. The necessity for discontinuity in both variables was pointed out by C. E. Wilder [4]. In [4] Wilder considered finding the adjoint of an $n$th order differential equation with multi-point boundary conditions. $\mathrm{He}$ also constructed the Green's function. The question of selfadjoint multi-point boundary value problems was considered by Mansfield [1]. Mansfield was interested in applications to the calculus of variations. He considered a vector system of differential equations with $n m$ boundary conditions where $n$ is the order of the system and $m$ is one more than the number of interior boundary points. It is then reduced to a vector system of order $\mathrm{nm}$ with no interior boundary points. The self-adjointness conditions are then found for the system of order $n \mathrm{~m}$ and then reinterpreted for the original system of order $n$. The results of the present paper seem to be more simply expressed. Some applications of multi-point boundary value problems with 
the need for possible discontinuities at interior boundary points are given in the paper by Weinberger [3].

In $\S 2$ we point out what seems to be an essential feature of Green's functions for multi-point boundary value problems, namely that intermediate boundary points impose discontinuities in the Green's function in one of its variables. This unsymmetrical discontinuity very effectively rules out hermitian symmetry. To restore hermitian symmetry it is necessary to have a symmetrical discontinuity in the Green's function in the other variable.

In $\S 3$ we consider a general vector differential equation with a multi-point boundary value problem. The differential equation is assumed to coincide with its Lagrange adjoint, and the Green's function is assumed to be hermitian symmetric with discontinuities on the lines $t=$ const. and $s=$ const. corresponding to the interior boundary points. The appropriate form for boundary conditions is then determined. If there are $m-1$ intermediate boundary points, it is found that the problem will require $m$ boundary conditions of a somewhat special form for self-adjointness.

In $\S 4$ we consider the case of a real second-order scalar differential equation with just one intermediate boundary point. In particular we consider the interesting case in which the discontinuity in the Green's function is only in the first derivative at $t=c$ and $s=c$ as well as at $t=s$, where $c$ is the interior boundary point.

In $\S 5$ we mention an application where multi-point boundary problems occur naturally and show that in the application given the self-adjointness conditions are fulfilled.

2. Discontinuities in Green's functions. In multi-point boundary value problems the presence of interior boundary points forces certain discontinuities in the Green's function. For self-adjointness it is necessary to impose similar discontinuities on the solutions. In this section we consider a simple case of a three-point boundary-value problem to see the nature of the imposed discontinuities.

Let us consider the problem:

$$
x^{\prime \prime}=g(t), \quad x(-1)=A x(0), \quad x(1)=B x(0) .
$$

It is not difficult to compute the Green's function for this problem. It is found that the solution is, if $A+B \neq 2$,

$$
x(t)=\int_{-1}^{1} G(t, s) g(s) d s
$$

where 


$$
\begin{aligned}
G(t, s) & =\frac{(1-B) t s+(A-1) t+(1-A-B) s-1}{2-A-B} \\
& =\frac{(1-B) t s+(1-B) t-s-1}{2-A-B}-1 \leqq s \leqq 0 \quad t \geqq s \\
& =\frac{(1-A) t s+(A-1) t+s-1}{2-A-B} \quad 0 \leqq s \leqq 1 \quad t \leqq s \\
& =\frac{(1-A) t s+(1-B) t+(A+B-1) s-1}{2-A-B} \\
& 0 \leqq s \leqq 1 \quad t \geqq s .
\end{aligned}
$$

Note that the Green's function $G(t, s)$ has to be given by four different formulas instead of the two required for a two-point boundary value problem. In this particular case, $G(t, s)$ is continuous for all $(t, s)$ in $-1 \leqq t, s \leqq 1$, but the derivative $G_{s}$ is discontinuous at $s=0$. It is also clear that hermitian symmetry occurs if and only if $A=$ $B=0$.

The boundary-value problem in the preceding paragraph can be written in the form

$$
L x=g(t)
$$

where $L$ is the differential operator which is generated by the differential expression $x^{\prime \prime}$ and which corresponds to the boundary conditions $x(-1)=A x(0), x(1)=B x(0)$. It is of interest to compute the adjoint of this operator, $L^{*}$. We can recover $L^{*}$ from the Green's function by writing the adjoint problem

$$
L^{*} x=h(t)
$$

in the form:

$$
x(t)=\int_{-1}^{1} \overline{G(s, t)} h(s) d s
$$

and then differentiating to obtain the differential expression generating $L^{*}$ and the associated boundary conditions. The integral expression is:

$$
\begin{aligned}
x(t)= & \int_{-1}^{t} \frac{t s(1-B)+t(1-A-B)+s(A-1)-1}{2-A-B} h(s) d s \\
& +\int_{t}^{1} \frac{t s(1-B)-t+s(1-B)-1}{2-A-B} h(s) d s \quad-1 \leqq t \leqq 0, \\
x(t)= & \int_{-1}^{t} \frac{t s(1-A)+t+s(A-1)-1}{2-A-B} h(s) d s
\end{aligned}
$$




$$
\begin{aligned}
+\int_{t}^{1} \frac{t s(1-A)+t(A+B-1)+s(1-B)-1}{2-A-B} h(s) d s & \\
& 0 \leqq t \leqq 1 .
\end{aligned}
$$

Differentiation gives:

$$
\begin{array}{rlr}
x^{\prime}(t)= & \int_{-1}^{t} \frac{s(1-B)+(1-A-B)}{2-A-B} h(s) d s & \\
& +\int_{t}^{1} \frac{s(1-B)-1}{2-A-B} h(s) d s & -1 \leqq t \leqq 0,
\end{array}
$$

and

$$
\begin{aligned}
x^{\prime}(t)= & \int_{-1}^{t} \frac{s(1-A)+1}{2-A-B} h(s) d s \\
& +\int_{t}^{1} \frac{s(1-A)+(A+B-1)}{2-A-B} h(s) d s \quad 0 \leqq t \leqq 1 .
\end{aligned}
$$

A second differentiation gives $x^{\prime \prime}=h(t)$, so that $L^{*}$ is generated by the same differential expression as $L$. This is natural, since $x^{\prime \prime}$ is its own Lagrange adjoint.

To find the boundary conditions of $L^{*}$ we set $t=-1,0-, 0+$, and 1 in the expressions for $x$ and $x^{\prime}$. We find:

$$
\begin{aligned}
x(-1) & =0 \\
x(0-) & =\int_{-1}^{0} \frac{s(A-1)-1}{2-A-B} h(s) d s+\int_{0}^{1} \frac{s(1-B)-1}{2-A-B} h(s) d s \\
x(0+) & =\int_{-1}^{0} \frac{s(A-1)-1}{2-A-B} h(s) d s+\int_{0}^{1} \frac{s(1-B)-1}{2-A-B} h(s) d s \\
x(1) & =0 \\
x^{\prime}(-1) & =\int_{-1}^{1} \frac{s(1-B)-1}{2-A-B} h(s) d s \\
x^{\prime}(0-) & =\int_{-1}^{0} \frac{s(1-B)+(1-A-B)}{2-A-B} h(s) d s+\int_{0}^{1} \frac{s(1-B)-1}{2-A-B} h(s) d s \\
x^{\prime}(0+) & =\int_{-1}^{0} \frac{s(1-A)+1}{2-A-B} h(s) d s+\int_{0}^{1} \frac{s(1-A)+(A+B-1)}{2-A-B} h(s) d s \\
x^{\prime}(1) & =\int_{-1}^{1} \frac{s(1-A)+1}{2-A-B} h(s) d s .
\end{aligned}
$$

There will be four boundary conditions, three of which are clearly

$$
x(-1)=0, \quad x(1)=0, \quad x(0-)=x(0+) .
$$

A fourth is

$$
x^{\prime}(0+)-x^{\prime}(0-)=B x^{\prime}(1)-A x^{\prime}(-1) .
$$


Note that the boundary conditions for $L^{*}$ impose a discontinuity on the derivative $x^{\prime}(t)$ at 0 . Note also that when $A=B=0$, two of the boundary conditions reduce to continuity of $x$ and $x^{\prime}$ at 0 while the other two are those of $L$ when $A=B=0$, so that again the self-adjointness of $L$ is verified when $A=B=0$.

Although the above is a special example, it is clear that in general intermediate boundary conditions will necessitate changes in formula of the Green's function at corresponding values of $s$. If the problem is to be self-adjoint, the Green's function must also have changes in formula at the corresponding values of $t$. In the next two sections we shall find just what boundary conditions will produce symmetric discontinuities in Green's functions and thus give selfadjoint multi-point boundary value problems.

3. Self-adjoint vector operators with multipoint boundary conditions. Let us consider an operator generated by the differential expression

$$
i x^{\prime}-A(t) x
$$

where the matrix $A(t)$ is continuous on $a \leqq t \leqq b$ and is hermitian symmetric. In this case, the differential expression coincides with its Lagrange adjoint.

Let $\Phi(t)$ be that fundamental matrix of the system $i x^{\prime}=A(t) x$ such that $\Phi\left(t_{0}\right)=I$ for some $t_{0}\left(a \leqq t_{0} \leqq b\right)$.

Lemma 1. The fundamental matrix $\Phi(t)$ is a unitary matrix for all $t$ in $a \leqq t \leqq b$.

Proof. Differentiate the identity

$$
\Phi(t) \Phi(t)^{-1}=I
$$

The result is

$$
\Phi^{\prime}(t) \Phi(t)^{-1}+\Phi(t) \Phi(t)^{-1^{\prime}}=0 .
$$

Using the fact that $i \Phi^{\prime}(t)=A(t) \Phi(t)$, we obtain

$$
\begin{gathered}
-i \Phi(t)^{-1^{\prime}}=\Phi(t)^{-1} A(t), \\
i \Phi(t)^{*-1,}=A^{*}(t) \Phi(t)^{*^{-1}}=A(t) \Phi(t)^{*^{-1}} .
\end{gathered}
$$

This shows that $\Phi(t)^{*^{-1}}$ is also a fundamental matrix of the equation $i x^{\prime}=A(t) x$. At $t=t_{0} \Phi(t)^{*^{-1}}=I^{*^{-1}}=I$. Since $\Phi(t)$ and $\Phi(t)^{*^{-1}}$ agree at $t=t_{0}$, they are identical. i.e.

$$
\Phi(t)^{*-1}=\Phi(t), \quad \Phi(t)^{*}=\Phi(t)^{-1} .
$$


This completes the proof.

To complete the definition of the differential operator we need to impose certain boundary conditions. We are interested in finding just what boundary conditions will make the operator self-adjoint. To find these, let us consider the inverse operator, which will be an integral operator with kernel the Green's function. Let us suppose that there are boundary conditions imposed at $t=a, t=b$, and $(m-1)$ interior points, so that they are imposed at:

$$
t=a=c_{0}<c_{1}<c_{2}<\cdots<c_{m-1}<c_{m}=b .
$$

As we have seen, we will have to have different formulas for the Green's function $G(t, s)$ in the $m^{2}+m$ cells into which the square $a \leqq t \leqq b, a \leqq s \leqq b$ is divided by the lines $t=c_{1}, s=c_{1}, \cdots, t=c_{m-1}$, $s=c_{m-1}, t=s$. In each such cell the Green's function will have the form

$$
\Phi(t) C \Phi(s)^{-1}=\Phi(t) C \Phi(s)^{*}
$$

where $C$ is a constant matrix. Moreover, at the diagonal $t=s$, the Green's function has a jump discontinuity given by:

$$
G(s+0, s)-G(s-0, s)=-i I .
$$

Finally, since we are interested in a self-adjoint operator, we must choose the various constant matrices so that for all $(t, s), G(t, s)=$ $G(s, t)^{*}$. The most general way of doing this is accomplished by the following.

Let the constant matrices $A_{i j}$ satisfy the relations $A_{i j}=A_{j i}^{*}$ $(i, j=1,2, \cdots, m)$. For $i \neq j$ let

$$
\begin{aligned}
& G(t, s)=\Phi(t) A_{i j} \Phi(s)^{*} \quad c_{i-1} \leqq t \leqq c_{i} \\
& c_{j-1} \leqq s \leqq c_{j} \text {. }
\end{aligned}
$$

For $i=j$, let

$$
\begin{aligned}
G(t, s) & =\Phi(t) A_{i i} \Phi(s)^{*}+\frac{1}{2} i \Phi(t) \Phi(s)^{*} \quad & c_{i-1} \leqq t \leqq s \leqq c_{i} \\
& =\Phi(t) A_{i i} \Phi(s)^{*}-\frac{1}{2} i \Phi(t) \Phi(s)^{*} \quad & c_{i-1} \leqq s \leqq t \leqq c_{i}
\end{aligned}
$$

The function $G(t, s)$ defined by the above is clearly hermitian symmetric, has the proper discontinuity when $t=s$, and also involves the fundamental matrix $\Phi(t)$ in the correct manner so that it is the Green's function of a differential operator. We proceed to find the boundary conditions generated by this Green's function. 
THEOREM 1. If $L$ is the differential operator generated by (3.1), whose inverse has the kernel $G(t, s)$ defined by (3.2) and (3.3), then $L$ is self-adjoint and is determined by the following $m$ boundary conditions:

$$
\begin{aligned}
\sum_{\substack{j=1 \\
j \neq i}}^{m} A_{i j}\left[u\left(c_{j}^{-}\right)\right. & \left.-u\left(c_{j-1}^{+}\right)\right]+\left(A_{i i}+\frac{1}{2} i I\right) u\left(c_{i}^{-}\right) \\
& -\left(A_{i i}-\frac{1}{2} i I\right) u\left(c_{i-1}^{+}\right)=0 \quad i=1,2, \cdots, m
\end{aligned}
$$

where $u(t)=\Phi(t)^{-1} x(t)$.

Proof. We first derive the boundary conditions (3.4) from the Green's function $G(t, s)$. This will show that the boundary conditions are necessary for self-adjointness. We then show that if $x(t)$ and $y(t)$ are differentiable functions such that (3.4) holds for both $x(t)$ and $y(t)$, then $(L x, y)=(x, L y)$. This will show that the boundary conditions (3.4) are sufficient for self-adjointness.

Suppose $x(t)$ is such that $L x=g(t)$. Then

$$
x(t)=\int_{a}^{b} G(t, s) g(s) d s,
$$

and we wish to show that $x(t)$ satisfies the conditions (3.4). If $c_{i-1} \leqq t \leqq c_{i}$, we find, using (3.2) and (3.3) that

$$
\begin{aligned}
u(t)= & \sum_{j=1}^{m} A_{i j} \int_{c_{j-1}}^{c_{j}} \Phi(s)^{*} g(s) d s-\frac{1}{2} i \int_{c_{i-1}}^{t} \Phi(s)^{*} g(s) d s \\
& +\frac{1}{2} i \int_{t}^{c_{i}} \Phi(s)^{*} g(s) d s .
\end{aligned}
$$

Hence:

$$
\begin{aligned}
u\left(c_{i}^{-}\right) & =\sum_{j=1}^{m} A_{i j} \int_{c_{j-1}}^{c_{j}} \Phi(s)^{*} g(s) d s-\frac{1}{2} i \int_{c_{i-1}}^{c_{i}} \Phi(s)^{*} g(s) d s \\
u\left(c_{i-1}^{+}\right) & =\sum_{j=1}^{m} A_{i j} \int_{c_{j-1}}^{c_{j}} \Phi(s)^{*} g(s) d s+\frac{1}{2} i \int_{c_{i-1}}^{c_{i}} \Phi(s)^{*} g(s) d s
\end{aligned}
$$

whence by subtraction:

$$
u\left(c_{i}^{-}\right)-u\left(c_{i-1}^{+}\right)=-i \int_{c_{i-1}}^{c_{i}} \Phi(s)^{*} g(s) d s .
$$

Thus the various integrals of $\Phi(s)^{*} g(s)$ are given by

$$
\int_{c_{i-1}}^{c_{i}} \Phi(s)^{*} g(s) d s=i\left[\left(u\left(c_{i}^{-}\right)-u\left(c_{i-1}^{+}\right)\right]\right.
$$


Using (3.6) in (3.5) we find

$$
u\left(c_{i}^{-}\right)=\sum_{j=1}^{m} A_{i j}(i)\left[u\left(c_{j}^{-}\right)-u\left(c_{j-1}^{+}\right)\right]-\frac{1}{2} i(i)\left[u\left(c_{i}^{-}\right)-u\left(c_{i-1}^{+}\right)\right] .
$$

Transposing and multiplying by $i$ we finally obtain:

$$
\sum_{j=1}^{m} A_{i j}\left[u\left(c_{j}^{-}\right)-u\left(c_{j-1}^{+}\right)\right]+\frac{1}{2} i u\left(c_{i}^{-}\right)+\frac{1}{2} i u\left(c_{i-1}^{+}\right)=0,
$$

which is equivalent to (3.4). This shows that in the case of selfadjointness, the boundary conditions (3.4) are satisfied.

We now wish to show that when $x(t)$ and $y(t)$ both satisfy (3.4), we have $(L x, y)-(x, L y)=0$. Now

$$
\begin{aligned}
(L x, y)-(x, L y)= & \int_{a}^{b} y^{*}(t)\left[i x^{\prime}(t)-A(t) x(t)\right] d t \\
& -\int_{a}^{b}\left[-i y^{* \prime}(t)-y^{*}(t) A^{*}(t)\right] x(t) d t \\
= & i \int_{a}^{b} \frac{d}{d t}\left\{y^{*}(t) x(t)\right\} d t
\end{aligned}
$$

since $A(t)=A^{*}(t)$. We therefore have to show that

$$
\sum_{j=1}^{m}\left\{y^{*}\left(c_{j}^{-}\right) x\left(c_{j}^{-}\right)-y^{*}\left(c_{j-1}^{+}\right) x\left(c_{j-1}^{+}\right)\right\}=0
$$

if $x$ and $y$ satisfy (3.4). To see this, use the notation $v(t)$ for $\Phi(t)^{-1} y(t)$. Then for each $j$ :

$$
\begin{aligned}
y^{*}\left(c_{j}^{-}\right) x\left(c_{j}^{-}\right)-y^{*}\left(c_{j-1}^{+}\right) x\left(c_{j-1}^{+}\right) \\
=v^{*}\left(c_{j}^{-}\right) u\left(c_{j}^{-}\right)-v^{*}\left(c_{j-1}^{+}\right) u\left(c_{j-1}^{+}\right) \\
=\frac{1}{2}\left\{\left[v^{*}\left(c_{j}^{-}\right)-v^{*}\left(c_{j-1}^{+}\right)\right]\left[u\left(c_{j}^{-}\right)+u\left(c_{j-1}^{+}\right)\right]\right. \\
\left.\quad+\left[v^{*}\left(c_{j}^{-}\right)+v^{*}\left(c_{j-1}^{+}\right)\right]\left[u\left(c_{j}^{-}\right)-u\left(c_{j-1}^{+}\right)\right]\right\} .
\end{aligned}
$$

Now use (3.4) which gives:

$$
\begin{aligned}
u\left(c_{j}^{-}\right)+u\left(c_{j-1}^{+}\right) & =2 i \sum_{k=1}^{m} A_{j k}\left[u\left(c_{k}^{-}\right)-u\left(c_{k-1}^{+}\right)\right] \\
v^{*}\left(c_{j}^{-}\right)+v^{*}\left(c_{j-1}^{+}\right) & \left.=-2 i \sum_{k=1}^{m} v^{*}\left(c_{k}^{-}\right)-v^{*}\left(c_{k-1}^{+}\right)\right] A_{j k}^{*} .
\end{aligned}
$$

Using (3.9) and (3.8) in (3.7) gives that the left member of (3.7) is equal to:

$$
\begin{array}{r}
i \sum_{j, k=1}^{m}\left[v^{*}\left(c_{j}^{-}\right)-v^{*}\left(c_{j-1}^{+}\right)\right] A_{j k}\left[u\left(c_{k}^{-}\right)-u\left(c_{k-1}^{+}\right)\right] \\
-i \sum_{j, k=1}^{m}\left[v^{*}\left(c_{k}^{-}\right)-v^{*}\left(c_{k-1}^{+}\right)\right] A_{j k}^{*}\left[u\left(c_{j}^{-}\right)-u\left(c_{j-1}^{+}\right)\right] .
\end{array}
$$


In the second sum in (3.10) interchange the summation indices and note that $A_{k j}^{*}=A_{j k}$. This proves that (3.10) is zero and hence that (3.7) holds. This completes the proof of Theorem 1.

4. Real second-order self-adjoint operators with three boundary points. In the present section we consider differential operators generated by the differential expression

$$
\left(p(t) x^{\prime}\right)^{\prime}+q(t) x \quad a \leqq t \leqq b
$$

where $p(t)$ is real and positive on $a \leqq t \leqq b$, and $p, p^{\prime}$, and $q$ are real and continuous on $a \leqq t \leqq b$. Here $x$ is a scalar-valued function. We wish to consider differential operators which are defined by boundary conditions at $t=a, t=b$, and at $t=c$, where $c$ lies between $a$ and $b$. We shall determine the form the boundary conditions must have in order that the differential operator be self-adjoint.

The procedure is as in $\S 3$. We set up the Green's function and require it to be hermitian symmetric, i.e. that $G(t, s)=\overline{G(s, t)}$. We then use the inverse (integral) operator to determine the boundary conditions on $x(t)$.

We consider only one intermediate boundary point. The case of several is very similar and leads to nothing essentially different. The important features of the problem are present with a single interior boundary point.

Let $t_{0}$ be an arbitrary point $a \leqq t_{0} \leqq b$, and let $\varphi(t)$ and $\psi(t)$ be those solutions of

$$
\left(p(t) x^{\prime}\right)^{\prime}+q(t) x=0
$$

such that

$$
\begin{aligned}
& \varphi\left(t_{0}\right)=p\left(t_{0}\right) \psi^{\prime}\left(t_{0}\right)=1, \\
& \psi\left(t_{0}\right)=p\left(t_{0}\right) \varphi^{\prime}\left(t_{0}\right)=0 .
\end{aligned}
$$

The expression

$$
p(t)\left[\varphi(t) \psi^{\prime}(t)-\psi(t) \varphi^{\prime}(t)\right]
$$

is identically constant, and is therefore identically +1 .

We now define the Green's function. The square $a \leqq t \leqq b, a \leqq$ $s \leqq b$ is divided into six cells by the lines $t=c, s=c$ and $t=s$. We must have $G(t, s)$ continuous for $t=s \neq c$ and the derivative $G_{t}(t, s)$ must have a discontinuity of $p(t)^{-1}$ as $t$ increases through $s$. We also choose $G(t, s)$ to be hermitian symmetric. These requirements lead to the following expressions for $G(t, s)$ : 


$$
\begin{aligned}
& G(t, s)= a_{11} \varphi(t) \varphi(s)+a_{12} \varphi(t) \psi(s)+a_{21} \psi(t) \varphi(s) \\
&+a_{22} \psi(t) \psi(s), \quad(a \leqq t \leqq c, c \leqq s \leqq b ; \\
&= b_{11} \varphi(t) \varphi(s)+b_{12} \varphi(t) \psi(s)+\overline{b_{12}} \psi(t) \varphi(s) \\
&+b_{22} \psi(t) \psi(s)+\frac{1}{2}[\varphi(t) \psi(s)-\psi(t) \varphi(s)], \\
& a \leqq t \leqq s \leqq c ; \\
&= b_{11} \varphi(t) \varphi(s)+b_{12} \varphi(t) \psi(s)+\overline{b_{12}} \psi(t) \varphi(s) \\
&+b_{22} \psi(t) \psi(s)-\frac{1}{2}[\varphi(t) \psi(s)-\psi(t) \varphi(s)] \\
& a \leqq s \leqq t \leqq c ; \\
&= c_{11} \varphi(t) \varphi(s)+c_{12} \varphi(t) \psi(s)+\overline{c_{12}} \psi(t) \varphi(s) \\
&+c_{22} \psi(t) \psi(s)+\frac{1}{2}[\varphi(t) \psi(s)-\psi(t) \varphi(s)], \\
& \quad c \leqq t \leqq s \leqq b ; \\
&= c_{11} \varphi(t) \varphi(s)+c_{12} \varphi(t) \psi(s)+\overline{c_{12}} \psi(t) \varphi(s) \\
&+c_{22} \psi(t) \psi(s)-\frac{1}{2}[\varphi(t) \psi(s)-\psi(t) \varphi(s)], \\
& c \leqq s \leqq t \leqq b ; \\
&= \overline{a_{11}} \varphi(t) \varphi(s)+\overline{a_{21}} \varphi(t) \psi(s)+\overline{a_{12}} \psi(t) \varphi(s) \\
&+\overline{a_{22}} \psi(t) \psi(s), \quad c \leqq t \leqq b, \quad a \leqq s \leqq c
\end{aligned}
$$

In (4.3) the coefficients $a_{11}, a_{12} \cdots c_{22}$ are arbitrary complex constants except that $b_{11}, b_{22}, c_{11}$, and $c_{22}$ are real.

THEOREM 2. The differential operator generated by (4.1) with associated Green's function (4.3) is self-adjoint. It is determined by the following boundary conditions:

$$
\begin{aligned}
& \frac{1}{2}\left[u(a)+u\left(c^{-}\right)\right]=B J\left[u\left(c^{-}\right)-u(a)++A J\left[u(b)-u\left(c^{+}\right)\right]\right. \\
& \frac{1}{2}\left[u\left(c^{+}\right)+u(b)\right]=A^{*} J\left[u\left(c^{-}\right)-u(a)\right]+C J\left[u(b)-u\left(c^{+}\right)\right]
\end{aligned}
$$

where

$$
\begin{array}{cc}
u(t)=\left(\begin{array}{c}
\varphi(t) \psi(t) \\
\varphi^{\prime}(t) \psi^{\prime}(t)
\end{array}\right)^{-1}\left(\begin{array}{l}
x(t) \\
x^{\prime}(t)
\end{array}\right), & A=\left(\begin{array}{ll}
a_{11} & a_{12} \\
a_{21} & a_{22}
\end{array}\right) \\
B=\left(\begin{array}{ll}
b_{11} & b_{12} \\
\overline{b_{12}} & b_{22}
\end{array}\right) \quad C=\left(\begin{array}{ll}
c_{11} & c_{12} \\
\bar{c}_{12} & c_{22}
\end{array}\right) & J=\left(\begin{array}{rr}
0 & 1 \\
-1 & 0
\end{array}\right) .
\end{array}
$$

Proof. The proof is entirely similar to the proof of Theorem 1 and will therefore not be given.

REMARK. Note that (4.4) is a set of four boundary conditions involving $x$ and $x^{\prime}$ at $t=a, t=c^{-}, t=c^{+}$and $t=b$.

A few further features should be noted. First if the matrix $A$ is the zero matrix, the two sets of boundary conditions are entirely uncoupled, and we actually have two separate self-adjoint problems, 
one on the interval $a \leqq t \leqq c$ and the other on the interval $c \leqq t \leqq b$.

If the point $c$ coincides with the endpoint $b$, then the problem is a two-point boundary-value problem with boundary condition:

$$
\frac{1}{2}[u(a)+u(b)]=B J[u(b)-u(a)]
$$

which can be shown to have the required form for self-adjoint twopoint boundary value problems.

An important special case of the question considered in this section is that in which $G(t, s)$ is continuous at $t=c$ and at $s=c$. This will mean that solutions of boundary-value problems of the form $L x=h(t)$ will not be discontinuous at $t=c$ (although the first derivative of such solutions may well be discontinuous at $t=c$ ).

Examination of (4.3) shows that $G(t, s)$ is continuous at $t=c$ and at $s=c$ if

$$
\left(B-A+\frac{1}{2} J\right)\left(\begin{array}{l}
\varphi(c) \\
\psi(c)
\end{array}\right)=\left(C-A^{*}-\frac{1}{2} J\right)\left(\begin{array}{l}
\varphi(c) \\
\psi(c)
\end{array}\right)=\left(\begin{array}{l}
0 \\
0
\end{array}\right) .
$$

For further analysis we assume that the point $t_{0}$ at which the solutions $\varphi(t)$ and $\psi(t)$ take on the values 1 and 0 is the point $c$. i.e. $\varphi(c)=p(t) \psi^{\prime}(c)=1, \varphi^{\prime}(c)=\psi(c)=0$. With this restriction, the condition for continuity of $G(t, s)$ at $t=c$ and at $s=c$ becomes that the first columns of the matrices $B-A+\frac{1}{2} J$ and $C-A^{*}-\frac{1}{2} J$ should be all zeros, which gives the relations:

$$
\begin{array}{ll}
b_{11}=a_{11}, & \overline{b_{12}}-a_{21}-\frac{1}{2}=0, \\
c_{11}=\overline{a_{11}}, & \overline{c_{12}}-\overline{a_{12}}+\frac{1}{2}=0 .
\end{array}
$$

This shows that $a_{11}$ is real and that $a_{11}=b_{11}=c_{11}$. Also $a_{12}=c_{12}+\frac{1}{2}$, $a_{21}=\overline{b_{12}}-\frac{1}{2}$. Thus the quantities $a_{22}, b_{11}, b_{12}, b_{22}, c_{12}$, and $c_{22}$ are still arbitrary, with $b_{11}, b_{22}, c_{22}$ real, $a_{22}, b_{12}, c_{12}$ allowed to be complex.

The boundary conditions (4.4) simplify somewhat in this case. Because of our choice of $\varphi$ and $\psi$, we find that

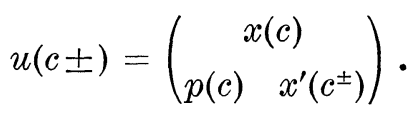

In the case of continuity of $G(t, s)$, the solution $x(t)$ is continuous at $t=c$, so we write $x(c)$ in place of $x\left(c^{ \pm}\right)$. Each of the equations in (4.4) represents two boundary conditions. When the relations (4.5) hold, the two equations corresponding to the first rows of the matrices in (4.4) are alike, so that we are left with just three independent boundary conditions. These can be written in the matrix expression 


$$
\begin{aligned}
& {\left[\begin{array}{cc}
-b_{12}+\frac{1}{2} & b_{11} \\
-b_{22} & \overline{b_{12}}+\frac{1}{2} \\
\overline{a_{22}} & -\overline{c_{12}}-\frac{1}{2}
\end{array}\right] u(a)+\left[\begin{array}{l}
b_{12}-c_{12} \\
b_{22}-a_{22} \\
c_{22}-\overline{a_{22}}
\end{array}\right] x(c)+} \\
& {\left[\begin{array}{c}
b_{11} \\
\overline{b_{12}}-\frac{1}{2} \\
-\overline{c_{12}}-\frac{1}{2}
\end{array}\right] p(c)\left[x^{\prime}\left(c^{+}\right)-x^{\prime}\left(c^{-}\right)\right]+\left[\begin{array}{cc}
c_{12}+\frac{1}{2} & -b_{11} \\
a_{22} & -\overline{b_{12}}+\frac{1}{2} \\
-c_{22} & \overline{c_{12}}-\frac{1}{2}
\end{array}\right] u(b)=0 .}
\end{aligned}
$$

Note that the derivatives $x^{\prime}\left(c^{-}\right)$and $x^{\prime}\left(c^{+}\right)$occur, in the case of continuous $G(t, s)$, only as the difference $x^{\prime}\left(c^{+}\right)-x^{\prime}\left(c^{-}\right)$. It is possible for the discontinuity in the derivative of the solution not to appear in the boundary conditions. However, this is true only under very restricted conditions. In fact, we must have $b_{11}=0, b_{12}=\frac{1}{2}$, and $c_{12}=-\frac{1}{2}$. If this is the case, the first boundary condition of the three in (4.6) becomes simply $x(c)=0$. The other two take the form

$$
\left[\begin{array}{cc}
-b_{22} & 1 \\
\overline{a_{22}} & 0
\end{array}\right] u(a)+\left[\begin{array}{cc}
a_{22} & 0 \\
-c_{22} & -1
\end{array}\right] u(b)=0 \text {. }
$$

As our later examples will show, solutions still will have discontinuous derivatives at $t=0$ even though the boundary conditions do not require this directly.

A second important special case is that in which two of a set of boundary conditions equivalent to (4.4) have the form

$$
A x(a)+B x^{\prime}(a)=0, \quad C x(b)+D x^{\prime}(b)=0 .
$$

If there is decoupling, so that the coefficients $a_{11}, a_{12}, a_{21}$, and $a_{22}$ all vanish, this leads to two self-adjoint problems one on $a \leqq t \leqq c$ with one boundary condition at $t=a$ and the other at $t=c$, and a second problem on $c \leqq t \leqq b$ with one boundary condition at $t=c$, and the other at $t=b$.

If decoupling is not present, the computation is somewhat involved, but it leads to the result that the second two boundary conditions have the form:

$$
\begin{aligned}
x\left(c^{+}\right) & =e^{i \theta}\left(p_{11} x\left(c^{-}\right)+p_{12} x^{\prime}\left(c^{-}\right)\right) \\
x^{\prime}\left(c^{+}\right) & =e^{i \theta}\left(p_{21} x\left(c^{-}\right)+p_{22} x^{\prime}\left(c^{-}\right)\right),
\end{aligned}
$$

where the $p_{i j}$ and $\theta$ are real, and $p_{11} p_{22}-p_{12} p_{21}=1$.

Suppose now that the above special cases are combined, so that $G(t, s)$ is continuous at $t=c$ and at $s=c$, and also two of the three resulting boundary conditions have the form (4.8). If there is decoupling, we always have the third boundary condition is $x(c)=0$. If there is not decoupling, the third boundary condition can be written in the form 


$$
x^{\prime}\left(c^{+}\right)-x^{\prime}\left(c^{-}\right)=A x(c),
$$

where $A$ is a real constant.

5. Examples. In the present section we give examples to illustrate the special cases mentioned in $\S 4$ together with some applications.

EXAmple 1. This is a case where $G(t, s)$ is continuous, but the boundary conditions are not separated. Because $G(t, s)$ is continuous, we will have three boundary conditions.

$$
L x=x^{\prime \prime}, \quad x(0)=0, \quad x(-1)=x^{\prime}(1)-x^{\prime}(-1)=x(1) .
$$

(The first boundary condition can be written as two: $x(0-)=0$, $x(0+)=0$.) The Green's function for this operator is:

$$
\begin{aligned}
G(t, s) & =t s & & 0 \leqq s \leqq 1,-1 \leqq t \leqq 0 \\
& =s & & -1 \leqq t \leqq s \leqq 0 \\
& =t & & -1 \leqq s \leqq t \leqq 0 \\
& =-t & & 0 \leqq t \leqq s \leqq 1 \\
& =-s & & 0 \leqq s \leqq t \leqq 1 \\
& =t s & & -1 \leqq s \leqq 0, \quad 0 \leqq t \leqq 1
\end{aligned}
$$

Since $G(t, s)$ is hermitian symmetric, the operator is self-adjoint. The eigenvalues and eigenfunctions of this operator come in two sets. One set of eigenvalues is the numbers $n^{2} \pi^{2}$ with eigenfunctions $\sin n \pi t$; the other is the roots of the equation $\tan \sqrt{\lambda}=2 \sqrt{\lambda}$, with eigenfunctions given by $(\operatorname{sgn} t) \sin \sqrt{\lambda} t$. Orthogonality and completeness of this set of eigenfunctions can be verified.

Although the boundary conditions do not require discontinuity at $t=0$, the second set of eigenfunctions all have discontinuous first derivatives at $t=0$.

ExAmple 2. This is a case where two of the boundary conditions are exclusively at one end, but no continuity of $G(t, s)$ is required.

$$
\begin{array}{ll}
L x=x^{\prime \prime} \quad x(-\pi)=0, \quad x(\pi)=0, \\
\\
x(0-)=\pi x(0+)+\left(\pi^{2}-1\right) x^{\prime}(0+) . \\
x^{\prime}(0-)=x(0+)+\pi x^{\prime}(0+) .
\end{array}
$$

The Green's function for this operator is: 


$$
\begin{aligned}
G(t, s) & =(t+\pi)(s-\pi) & & 0 \leqq s \leqq \pi,-\pi \leqq t \leqq 0, \\
& =-(t+\pi) & & -\pi \leqq t \leqq s \leqq 0, \\
& =-(s+\pi) & & -\pi \leqq s \leqq t \leqq 0, \\
& =(s-\pi) & & 0 \leqq t \leqq s \leqq \pi, \\
& =(t-\pi) & & 0 \leqq s \leqq t \leqq \pi, \\
& =(t-\pi)(s+\pi) & & 0 \leqq t \leqq \pi, \quad-\pi \leqq s \leqq 0
\end{aligned}
$$

Since $G(t, s)$ is hermitian symmetric, the operator is self-adjoint, Again the eigenvalues and eigenfunctions come in two sets. One set of eigenvalues is the roots of the equation $\tan \sqrt{\lambda} \pi=(\pi-1) \sqrt{\lambda}$ (which includes one negative eigenvalue) with eigenfunctions $\sin \sqrt{\lambda}(t-\pi \operatorname{sgn} t)$; the other set is the roots of the equation $\tan \sqrt{\lambda} \pi=(\pi+1) \sqrt{\bar{\lambda}}$ with eigenfunctions $\operatorname{sgn} t \sin \sqrt{\lambda}(t-\pi \operatorname{sgn} t)$. The first set of eigenfunctions have a discontinuity at $t=0$; the second set of eigenfunctions are continuous at $t=0$, but the derivatives have a discontinuity.

EXAMPLE 3. This example combines the features of the two preceding examples.

$$
L x=x^{\prime \prime}, \quad x(-1)=0, \quad x(1)=0, \quad x^{\prime}(0+)-x^{\prime}(0-)=-x(0) .
$$

(A fourth boundary condition could be written: $x(0-)=x(0+)$.) The Green's function for this operator is

$$
\begin{aligned}
G(t, s) & =(t+1)(s-1) & & -1 \leqq t \leqq 0,0 \leqq s \leqq 1, \\
& =-(t+1) & & -1 \leqq t \leqq s \leqq 0, \\
& =-(s+1) & & -1 \leqq s \leqq t \leqq 0, \\
& =(s-1) & & 0 \leqq t \leqq s \leqq 1, \\
& =(t-1) & & 0 \leqq s \leqq t \leqq 1, \\
& =(t-1)(s+1) & & 0 \leqq t \leqq 1, \quad-1 \leqq s \leqq 0 .
\end{aligned}
$$

Since $G(t, s)$ is hermitian symmetric, the operator is self-adjoint. Again there are two sets of eigenvalues and eigenfunctions. One set is the numbers $n^{2} \pi^{2}$ with eigenfunctions $\sin n \pi t$; the second set is the roots of the equation $\tan \sqrt{\lambda}=2 \sqrt{\lambda}$, with eigenfunctions

$$
\operatorname{sgn} t \sin \{\sqrt{\lambda}(t-\operatorname{sgn} t)\} \text {. }
$$

The eigenfunctions of the second set have discontinuous derivatives at $t=0$.

The third example is related to an application. Consider a string of length 2 extending from $x=-1$ to $x=1$, and let there be a point mass at $x=0$. The velocity of propagation of waves along the 
string is determined from the tension and linear density of the string and, if this velocity is $v$, the transverse displacements of the string satisfy the equation

$$
y_{x x}=\frac{1}{v^{2}} y_{t t}
$$

The boundary conditions imposed on $y(x, t)$ are: $y(-1, t)=y(1, t)=0$, and to account for the point mass

$$
m y_{t t}(0, t)=\text { const. }\left(y_{x}(0+, t)-y_{x}(0-, t)\right. \text {. }
$$

Thus if the mass $m$ is positive, there must be a discontinuity in $y_{x}$ at $s=0$.

This problem leads to an eigenvalue problem when variables are separated, and in this particular case we find:

$$
\begin{gathered}
\frac{d^{2} X}{d x^{2}}+\lambda X=0, \quad X(-1)=X(1)=0, \\
X^{\prime}(0+)-X^{\prime}(0-)=\text { const. } X(0),
\end{gathered}
$$

which is similar to Example 3. Since this is a self-adjoint boundary problem, the eigenfunctions (which correspond to the normal modes of vibration) will be a complete orthonormal set so that eigenfunction expansions can be used to obtain the solution $y(x, t)$.

In a similar manner other vibrating string problems with point masses and vibrating beam problems with point loadings lead to multipoint boundary-value problems which are self-adjoint.

\section{REFERENCES}

1. R. Mansfield, Differential systems involving k-point boundary conditions, in Contributions to the Calculus of Variations 1938-1914, Chicago, 1942, 413-452.

2. J. W. Neuberger, The lack of self-adjointness in three-point boundary-value problems, Pacific J. Math. (to appear)

3. H. Weinberger, An extension of the classical Sturm-Liouville theory, Duke, Math. J. 22 (1955), 1-14.

4. C. E. Wilder, Problems in the theory of linear differential equations with auxiliary conditions at more than two points, Trans. Amer. Math. Soc. 19 (1918), 157-166.

5. A. Zettl, The lack of self-adjointness in three point boundary-value problems, Proc. Amer. Math. Soc. 17 (1966), 368-371.

Received July 29, 1966. 



\title{
PACIFIC JOURNAL OF MATHEMATICS
}

\author{
EDITORS
}

\section{H. ROYDEN}

Stanford University

Stanford, California

\section{J. P. JANS}

University of Washington Seattle, Washington 98105

\section{J. DugundJI}

Department of Mathematics

Rice University

Houston, Texas 77001

RICHARD ARENS

University of California

Los Angeles, California 90024

\section{ASSOCIATE EDITORS}

E. F. BECKENBACH
F. WOLF

K. YosidA

\section{SUPPORTING INSTITUTIONS}

\author{
UNIVERSITY OF BRITISH COLUMBIA \\ CALIFORNIA INSTITUTE OF TECHNOLOGY \\ UNIVERSITY OF CALIFORNIA \\ MONTANA STATE UNIVERSITY \\ UNIVERSITY OF NEVADA \\ NEW MEXICO STATE UNIVERSITY \\ OREGON STATE UNIVERSITY \\ UNIVERSITY OF OREGON \\ OSAKA UNIVERSITY \\ UNIVERSITY OF SOUTHERN CALIFORNIA
}

\author{
STANFORD UNIVERSITY \\ UNIVERSITY OF TOKYO \\ UNIVERSITY OF UTAH \\ WASHINGTON STATE UNIVERSITY \\ UNIVERSITY OF WASHINGTON \\ MERICAN MATHEMATICAL SOCIETY \\ CHEVRON RESEARCH CORPORATION \\ TRW SYSTEMS \\ NAVAL ORDNANCE TEST STATION
}

Mathematical papers intended for publication in the Pacific Journal of Mathematics should be in typed form or offset-reproduced, double spaced with large margins. Underline Greek letters in red, German in green, and script in blue. The first paragraph or two must be capable of being used separately as a synopsis of the entire paper. It should not contain references to the bibliography. Manuscripts may be sent to any one of the four editors. All other communications to the editors should be addressed to the managing editor, Richard Arens, University of California, Los Angeles, California 90024.

Each author of each article receives 50 reprints free of charge; additional copies may be obtained at cost in multiples of 50 .

The Pacific Journal of Mathematics is published monthly. Effective with Volume 16 the price per volume (3 numbers) is $\$ 8.00$; single issues, $\$ 3.00$. Special price for current issues to individual faculty members of supporting institutions and to individual members of the American Mathematical Society: $\$ 4.00$ per volume; single issues $\$ 1.50$. Back numbers are available.

Subscriptions, orders for back numbers, and changes of address should be sent to Pacific Journal of Mathematics, 103 Highland Boulevard, Berkeley 8, California.

Printed at Kokusai Bunken Insatsusha (International Academic Printing Co., Ltd.), 7-17, Fujimi 2-chome, Chiyoda-ku, Tokyo, Japan.

PUBLISHED BY PACIFIC JOURNAL OF MATHEMATICS, A NON-PROFIT CORPORATION

The Supporting Institutions listed above contribute to the cost of publication of this Journal, but they are not owners of publishers and have no responsibility for its content or policies. 


\section{Pacific Journal of Mathematics}

\section{Vol. 24, No. $2 \quad$ June, 1968}

John Suemper Alin and Spencer Ernest Dickson, Goldie's torsion theory and its derived functor . . . . . . . . . . . . . . . . . . . . . . . . . . . 195

Steve Armentrout, Lloyd Lesley Lininger and Donald Vern Meyer,

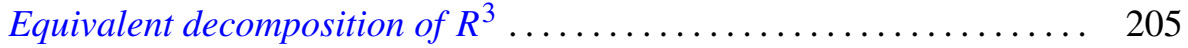

James Harvey Carruth, A note on partially ordered compacta ........... 229

Charles E. Clark and Carl Eberhart, A characterization of compact

connected planar lattices........................... 233

Allan Clark and Larry Smith, The rational homotopy of a wedge ......... 241

Donald Brooks Coleman, Semigroup algebras that are group algebras .... 247

John Eric Gilbert, Convolution operators on $L^{p}(G)$ and properties of

locally compact groups ............................. 257

Fletcher Gross, Groups admitting a fixed-point-free automorphism of order

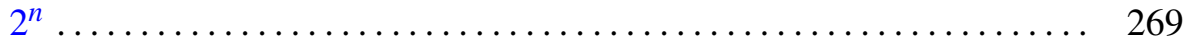

Jack Hardy and Howard E. Lacey, Extensions of regular Borel measures . . . 277

R. G. Huffstutler and Frederick Max Stein, The approximation solution of

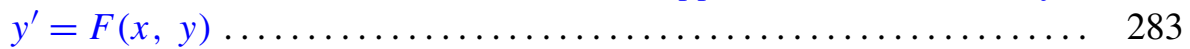

Michael Joseph Kascic, Jr., Polynomials in linear relations . . . . . . . . . . 291

Alan G. Konheim and Benjamin Weiss, A note on functions which

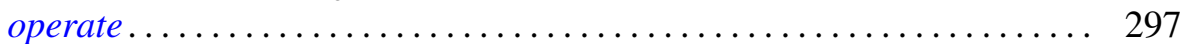

Warren Simms Loud, Self-adjoint multi-point boundary value problems ... 303 Kenneth Derwood Magill, Jr., Topological spaces determined by left ideals

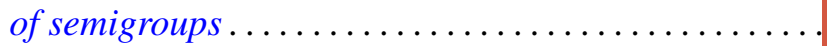

Morris Marden, On the derivative of canonical products . . .

J. L. Nelson, A stability theorem for a third order nonlinear differential

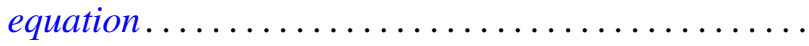

Raymond Moos Redheffer, Functions with real poles and zeros ...

Donald Zane Spicer, Group algebras of vector-valued functions ...

Myles Tierney, Some applications of a property of the functor $E f$ 